\title{
Nonsteroidal Anti-Inflammatory Drug Hypersensitivity in Preschool Children
}

\author{
Mona Iancovici Kidon, Liew Woei Kang, Chiang Wen Chin, Lim Siok Hoon, and Van Bever Hugo
}

\begin{abstract}
Although extensively studied in adults, nonsteroidal anti-inflammatory drug (NSAID) hypersensitivity in children, especially in young children, remains poorly defined. Pediatricians, prescribing antipyretics for children, rarely encounter significant problems, but the few epidemiologic studies performed show conflicting results. Although it is clear that some patients with acetylsalicylic acid (ASA)sensitive asthma have their clinical onset of disease in childhood and bronchoconstriction after ASA challenge is seen in 0 to $22 \%$ of asthmatic children so challenged, ibuprofen at antipyretic doses may cause acute respiratory problems only in a very small number of mild to moderate asthmatics. The recently elucidated mechanism of action of acetaminophen may explain some occurrences of adverse reactions in patients with cross-reactive NSAID hypersensitivity on the basis of its inhibitory activity on the newly described enzyme, cyclooxygenase (COX)-3. This nonspecific sensitivity to inhibition of COX is most likely genetically determined and shows a remarkable association with atopic disease even in the very young age group and possibly an increased predilection in specific ethnic groups. This review summarizes state-of-the-art published data on NSAID hypersensitivity in preschool children.
\end{abstract}

Key words: acetaminophen, acetylsalicylic acid (ASA), children, hypersensitivity, ibuprofen, NSAID, preschool

A cetylsalicylic acid (ASA) and other nonsteroidal antiinflammatory drugs (NSAIDs) are a group of medications with heterogenic chemical structures, sharing the capability of inhibiting with various degrees of specificity and efficacy the cyclooxygenase (COX) enzymes responsible for the prostaglandin synthetase pathway of arachidonic acid metabolism. This blockade also results in the shunting of arachidonic acid toward the 5-lipoxigenase pathway, resulting in increased production and release of cysteinyl leukotrienes.

Although extensively studied in adults, NSAID hypersensitivity in children, especially in young children, remains a poorly defined area in both its clinical and epidemiologic aspects. ASA and NSAIDs are not widely used in this group of children, secondary to both the recognized association of ASA use and Reye syndrome ${ }^{1}$ and the absence of appropriate preparations or studied

Mona Iancovici Kidon: Rheumatology, Immunology and Allergy Service, Department of Paediatric Medicine, KK Children's Hospital, Singapore; Liew Woei Kang, Chiang Wen Chin, and Lim Siok Hoon: Department of Paediatric Medicine, KK Children's Hospital, Singapore; and Van Bever Hugo: Department of Paediatrics, National University Hospital, Singapore.

Correspondence to: Dr. Mona Iancovici Kidon, 100 Bukit Timah Road, 229899 Singapore; e-mail: Mona.Kidon@kkh.com.sg.

DOI $10.2310 / 7480.2007 .00008$ indications for most other NSAIDs in infants and toddlers. The only extensively used preparation is ibuprofen, a propionic acid derivative and a nonspecific inhibitor of COX-1 (mainly) and COX-2, available since the early 1990s in appropriate formulary and approved for "overthe-counter" ${ }^{2}$ use for fever and acute pain at this age.

Acetaminophen, although not usually considered an NSAID medication, is the most ubiquitously used antipyretic medication in children and is included in this review of hypersensitivity reactions in small children for the reasons outlined below. An "old" medication whose mechanism of action was recently defined, ${ }^{3}$ it has no significant effect on peripheral COX-1 and COX-2. Its antipyretic effect is consistent with a central nervous system-mediated activity on a newly defined COX enzyme, COX-3, found only in the brain and spinal cord. This selective inhibition of COX-3 mediates the effect of acetaminophen in relieving pain and reducing fever without unwanted gastrointestinal side effects. Thus, although having almost no anti-inflammatory effects, even at high doses, so, strictly speaking, it is not an NSAID medication, acetaminophen, like ASA and the NSAID, is an inhibitor of prostaglandin synthesis.

Hypersensitivity reactions to ASA and NSAIDs fall largely into two major groups according to their putative pathophysiologic mechanisms and the specificity of the inciting medication. ${ }^{4}$ The first group comprises nonspe- 
cific reactions (usually cross-reactive with other NSAIDs), with severity most likely related to the COX inhibitor activity of the inciting medication. This group is further subdivided into four related syndromes according to their clinical features and includes (1) NSAID-induced asthma and rhinitis in asthmatic patients, previously dubbed ASAexacerbated respiratory disease (AERD) or the "ASA triad"; (2) NSAID-induced urticaria/angioedema in patients with chronic urticaria; (3) ASA- or NSAIDinduced cross-reacting urticaria in otherwise normal individuals; and (4) blended reactions in otherwise normal individuals.

The second group contains drug-specific, most likely immunologically mediated, reactions. This group is subdivided into four related categories, with reactions being specific to one inciting medication or one chemically related group only, and the clinical presentations are reminiscent of at least one classic Gel and Coombs classification of immunologic reaction types: (5) single NSAID-induced urticaria/angioedema in otherwise normal subjects (immediate-type, isolated skin reactions); (6) single NSAID-induced anaphylaxis and anaphylactoid syndromes (immediate-type, systemic reactions); (7) aseptic meningitis caused by a specific NSAID; and (8) hypersensitivity pneumonitis caused by a specific NSAID.

The above-described heterogeneous clinical presentation patterns and different putative etiologic mechanisms underlie various recommended diagnostic challenge protocols in the adult literature specific for each clinical presentation.

\section{Objective}

The objective of this study was to ascertain whether the above classification can accurately describe published data on hypersensitivity reactions to ASA, NSAID, and acetaminophen in preschool children and to summarize the available published data on NSAID hypersensitivity in this age group.

\section{Methods}

We conducted a review of English-language publications extracted from the PubMed database, from 1980 to November 2005, using the key words aspirin, ASA, ibuprofen, acetaminophen, paracetamol, nonsteroidal, NSAID, hypersensitivity, infant, toddler, preschool, and child. All extracted citations were manually reviewed for the inclusion of patients aged 6 years and younger. Additional candidate publications were extracted from the relevant citations and previously published general reviews of NSAID hypersensitivity in children. Data from any publications that included information on young children were used for the purpose of this review.

\section{Results}

Two hundred sixty-seven publications fulfilled the initial search criteria. On closer inspection, only 72 publications included any patients in our targeted age group. From these, 12 were reviews of previously published data, mainly excluded from the review. Sixty publications were included, mostly small case series or nonrandomized trials. Fourteen were single case reports, and only four publications summarized data from randomized controlled prospective studies, looking at any aspect of adverse drug reactions in childhood and incorporating data on preschoolers. For a summary of included and excluded publications, see Figure 1.

\section{Epidemiology}

The available epidemiologic data are summarized in Table 1. Only one identified study addressed the issue of the prevalence of hypersensitivity reactions to ASA in the general population, including young children. ${ }^{5}$ It is notable that patients with a history of chronic pulmonary disease, recurrent rhinitis, or recurrent urticaria were excluded from this study. The questionnaire-derived frequency in this "normal" population was $0.3 \%$ (6 of $1,974)$ in adults and $0.32 \%$ ( 2 of 618 ) in children. The two documented cases in the pediatric age range complained of urticaria and were less than 6 years of age.

One prospective study looked at hospital admissions in children less than 2 years of age: ${ }^{6} 4.3 \%$ of general admissions were due to a suspected adverse drug reaction. Four hospitalizations $(0.8 \% ; 4$ of 512$)$ were due to pharmaceutical combinations that included ASA; however, two of eight $(25 \%)$ reactions classified as severe were due to this same drug combination. Hypersensitivity was not proven in any of these reported cases.

The prevalence of self-reported NSAID hypersensitivity in inpatients from a general pediatric hospital was estimated to be $0.5 \%$ (19\% of a reported $2.6 \%$ for all drug hypersensitivities) in the KK Children's Hospital in Singapore. ${ }^{7}$

In a series of children from the Royal Children's Hospital in Parkville, Australia, who developed adverse reactions to NSAID during their hospitalization, ${ }^{8} 8$ of 25 $(32 \%)$ were children below 6 years of age. The documented 


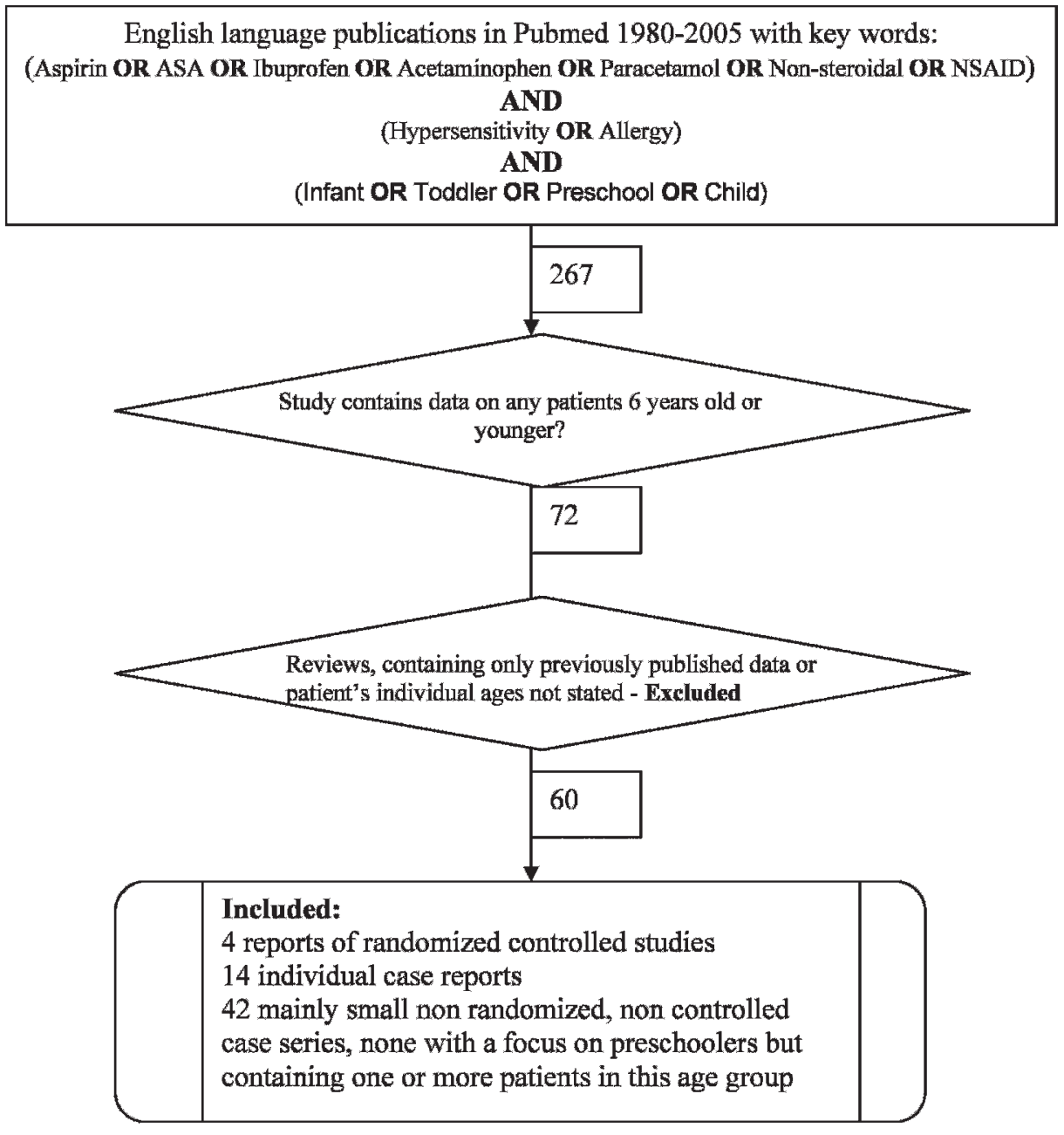

Figure 1. Diagram of inclusion and exclusion criteria of publications for the present review.

Table 1. Summary of Main Epidemiologic Data

\begin{tabular}{l}
\hline References \\
In the general population, the incidence of NSAID hypersensitivity in young children is low, although it may \\
equal that found in healthy adults \\
ADRs account for only $4.3 \%$ of general pediatric hospitalizations, but ASA-containing medications constitute \\
approximately one-fifth of these and tend to cause clinically severe reactions \\
About a third of children developing acute NSAID hypersensitivity in a medical setting are 6 years old or younger \\
Atopy and allergic disease are the most significant risk factors for the development of NSAID hypersensitivity in \\
young children (and older ones) \\
The prevalence of NSAID hypersensitivity in atopic children is $2 \%$ but lower in the young age group and increases \\
with age \\
The incidence of challenge-derived ASA hypersensitivity in asthmatics depends on the diagnostic protocol used \\
In the young age group, there is no female preponderance (like that seen in adults with ASA-sensitive asthma) \\
Antipyretic doses of ibuprofen in young children do not seem to increase the risk of hospitalization due to asthma \\
or bronchitis compared with acetaminophen \\
The risk of acute exacerbations in young asthmatic children during an acute illness is not increased by the use \\
of antipyretic doses of ibuprofen \\
\hline
\end{tabular}

$\mathrm{ADR}=$ adverse drug reaction; ASA $=$ acetylsalicylic acid; NSAID = nonsteroidal anti-inflammatory drug. 
cases run the spectrum of rash, facial angioedema, wheezing, and anaphylactic or anaphylactoid reactions.

In selected groups of children, challenge-derived incidence of ASA hypersensitivity was estimated to be between 0 and $28 \%$ in children with asthma, most of whom were older than 6 years of age, ${ }^{9-12}$ and $2 \%$ in young atopic children attending a general allergy clinic. ${ }^{13}$ An increased rate of NSAID hypersensitivity was observed with increasing age in this group.

Atopy seems to be a significant risk factor for ASA and NSAID hypersensitivity reactions in general ${ }^{14}$ and a significant risk factor for such reactions in young children. In the series of Rachelefsky and colleagues, ${ }^{11}$ with an incidence of $28 \%$ positive challenge responses to oral ASA in asthmatic, mostly older children, all 50 patients enrolled had at least one positive skin-prick test and associated allergic rhinitis. In our published group of NSAID-hypersensitive Asian children, ${ }^{15}$ with $25 \%$ of the patients being less than 6 years of age, $89 \%$ had a positive skin-prick test and/or evidence of clinically relevant atopic disease.

In the pediatric age group, as opposed to the published data in adult series, there is no female preponderance in patients with NSAID hypersensitivity. In a prospective case series published by Speer and colleagues, ${ }^{16} 10$ of 171 patients had onset of hypersensitivity before 5 years of age-six males and four females. The earliest age was 12 months. Male preponderance was also seen by our group, with the youngest reported onset of reactions at 3 months. ${ }^{15}$

The best quality of epidemiologic data available for this age group comes from two relatively recent publications summarizing data from a large randomized controlled study. ${ }^{17,18}$ The first focused on the safety profile of acetaminophen and ibuprofen at antipyretic doses for the treatment of an acute febrile illness in children younger than 2 years of age. More than 27,000 children were enrolled with a median age of 13 months (range 1-23 months). There were no hospitalizations secondary to acute anaphylaxis. The risk of hospitalization with a diagnosis of asthma or bronchitis was 24 in 10,000. The relative risk of asthma or bronchitis hospitalization with ibuprofen compared with acetaminophen was 0.9. The study did not include data on minor adverse events such as rashes not requiring hospitalization.

The second evaluated the safety of ibuprofen at antipyretic doses in asthmatic children 6 months to 12 years of age. In this study, 1,879 asthmatic patients, with a median age of 46.3 months, were randomized to receive acetaminophen $12 \mathrm{mg} / \mathrm{kg}$, ibuprofen $5 \mathrm{mg} / \mathrm{kg}$, and ibuprofen $10 \mathrm{mg} / \mathrm{kg}$ as antipyretic treatment. The rate of hospitalization with asthma overall was 96 per 10,000.
There was no statistically significant difference between the groups. The rate of outpatient visits for asthma was 335 per 10,000 courses of therapy (3.4\%). A small but statistically significant protective effect was observed for the ibuprofen treatment, especially in those patients for whom the initial diagnosis was an acute respiratory infection.

Both publications report data stemming from the Boston University Fever Study, so both excluded from enrollment children with any known sensitivity to acetaminophen, ibuprofen, ASA, or any other NSAID, as well as children with all or part of the syndrome of nasal polyps, angioedema, and bronchospastic reactivity to ASA or an NSAID. Therefore, they likely underestimate the true prevalence of the problem, even though accurately reporting the incidence of acute reactions in healthy and asthmatic young children without known previous reactions.

\section{Etiology and Pathophysiology}

Although the pathophysiology of drug-specific immunemediated reactions is mostly well understood, the mechanism of the cross-reactive reactions seen with the NSAID is still somewhat obscure. Although abnormalities in the leukotriene pathway of arachidonic acid metabolism have been postulated by multiple investigators, ${ }^{19-22}$ and leukotriene receptor antagonists seem to be effective in the treatment of AERD, ${ }^{23,24}$ this has not been shown in all populations examined. ${ }^{25,26}$ Clinical data from in vivo provocation testing of adult asthmatics have failed to show consistent significant differences in leukotriene urinary excretion between ASA-sensitive and nonsensitive individuals. $^{27}$ Genetic analyses in different groups of patients also involve abnormalities in the TBX21 gene and interferon- $\gamma$ production, ${ }^{28}$ in the DR/DP haplotypes associated with antigen presentation, ${ }^{29,30}$ and even a viral etiology has been postulated in adults. ${ }^{31}$ No genetic association studies have been published so far in children, and the mechanisms of ASA-associated angioedema and urticaria, the most prevalent form of NSAID hypersensitivity in this age group, are still speculative.

\section{Clinical Presentation}

The available clinical presentation and symptomatology data are summarized in Table 2. At the level of case reports or specific cases within reported case series, we have found documentation of the following clinical presentations in children less than 6 years of age: in the group of nonspecific, cross-reactive, most likely COX inhibitor- 
Table 2. Summary of Clinical Presentations of NSAID Hypersensitivity in Preschool Children

References

Nonspecific, cross-reactive, COX inhibitor related

ASA-exacerbated respiratory disease (AERD)

9-12, 32

Angioedema/urticaria in children with chronic urticaria

$33-37$

Angioedema/urticaria in children without chronic urticaria

$15,38-40$

Mixed reactions (angioedema/urticaria and acute respiratory symptoms, bronchospasm)

15,41

The most common clinical manifestation of NSAID hypersensitivity at this age is facial angioedema with or without

13,15 generalized urticaria

Drug-specific, immune-mediated reactions

Immediate single drug-mediated urticaria/angioedema

38,42

Delayed-type hypersensitivity reactions (eg, fixed drug eruptions and toxic epidermal necrolysis)

$38,43-46$

No publications of ASA-/NSAID-induced aseptic meningitis or hypersensitivity pneumonitis were identified

ASA $=$ acetylsalicylic acid; COX $=$ cyclooxygenase; NSAID $=$ nonsteroidal anti-inflammatory drug.

dependent reactions, cases of cross-reactive AERD, ${ }^{9-12,32}$ cross-reactive angioedema/urticaria in children with chronic urticaria, ${ }^{33-37}$ cross-reactive angioedema/urticaria in children without chronic urticaria ${ }^{15,38-40}$ and mixed reactions, i.e. angioedema/urticaria and acute respiratory symptoms, bronchospasm has been documented. ${ }^{15,41}$

Overall, the most common clinical manifestation of NSAID hypersensitivity at this age is facial angioedema with or without generalized urticaria. A classic example of facial angioedema and urticaria provoked by ibuprofen in a young child is depicted in Figure 2 .

In the group of drug-specific, most likely immunemediated reactions, published cases were found detailing immediate single drug-mediated urticaria/angioedema ${ }^{38,42}$ and cases of delayed-type hypersensitivity reactions, that is, fixed drug eruptions and toxic epidermal necrolysis. ${ }^{38,43-46}$ No publications of ASA- or NSAID-induced aseptic meningitis or hypersensitivity pneumonitis were identified in the studied age group.

\section{Diagnosis}

In the absence of laboratory or other available diagnostic tests, the gold standard for the diagnosis of NSAID hypersensitivity in children and in adults is the drug provocation test. All drug provocation tests should strictly adhere to the general principles of patient safety and patient beneficence outlined in published guidelines. ${ }^{47}$ All challenge procedures should be performed by trained personnel in a safe and well-appointed environment, adequately prepared for the treatment of life-threatening allergic reactions.

Oral and inhalation challenge protocols are published and seem widely accepted in adults, although significant variations between research centres and practitioners abound. Protocols for challenge, as summarized in Middleton's chapter on ASA hypersensitivity, vary also according to the clinical presentation and the putative mechanism of action. ${ }^{4}$ In the article by Rachelefsky and colleagues ${ }^{11}$ investigating AERD in asthmatic children 6 to 18 years old, $300 \mathrm{mg}$ of ASA or $100 \mathrm{mg}$ of placebo was given on two separate days. Fourteen of 50 children $(28 \%)$ responded with more than a 30\% decrease in their pulmonary function tests after ASA ingestion, on average worse at 4 hours after challenge than at 30 minutes. Eleven of these 14 patients complained of continuing symptoms for the next 24 hours after challenge. This and similar publications support the use of a prolonged (over several

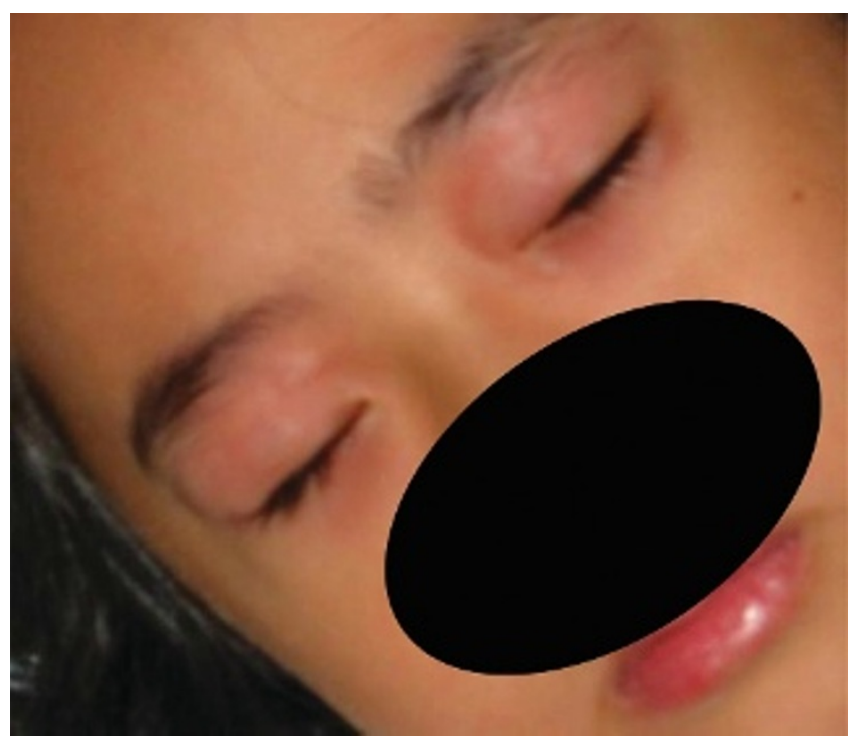

Figure 2. Periorbital urticarial lesions and lower lip angioedema in a 5 -year-old girl after oral provocation with $5 \mathrm{mg} / \mathrm{kg}$ of ibuprofen. 
days), incremental challenge in pediatric asthmatics with suspected AERD, similar to the proposed protocol in adults.

For nonasthmatic children with angioedema-urticaria or mixed-type reactions, a single challenge dose of $100 \mathrm{mg}$ of ibuprofen or ASA and $180 \mathrm{mg}$ of acetaminophen has been in use by Sanchez Borges and colleagues for children more than 8 years of age. ${ }^{14}$ Botey and colleagues ${ }^{37}$ used a 1 $\mathrm{mg}$ challenge of ASA on day 1 followed by $150 \mathrm{mg}$ on day 3 if no reactions occurred in their series of patients with chronic angioedema/urticaria and ASA sensitivity. Six of nine children in this series were younger than 6 years of age; the two youngest (aged 2 and 3 years, respectively) reacted to the first $1 \mathrm{mg}$ dose.

A classic protocol of doubling concentrations every 30 minutes is recommended in normal adults without evidence of chronic urticaria, similar to drug provocation protocols in suspected immediate-type reactions. ${ }^{4}$ An alternative drug challenge protocol was adopted by our group $^{15,41}$ secondary to severe respiratory reactions developing after 4 to 6 hours of challenge with the classic doubling protocol in a group of young children with reported urticaria, angioedema, and mixed reactions. We are currently using a protocol of $2.5 \mathrm{mg} / \mathrm{kg}$ of body weight of either ASA or ibuprofen or $5 \mathrm{mg} / \mathrm{kg}$ of acetaminophen ingested at hourly intervals to a maximal dose of $10 \mathrm{mg} / \mathrm{kg}$ and $20 \mathrm{mg} / \mathrm{kg}$, respectively, or until a positive reaction is elicited (Table 3 ). This protocol seems to be most sensitive to young children as it takes into account the significant variation in weight between ages 1 and 10. Patients are monitored at least 2 hours after the last challenge dose or, if a reaction occurs, until it clinically subsides. We have not observed any late-phase reactions even in asthmatic children after undergoing this challenge protocol.

\section{Prognosis}

A single published long-term follow-up of patients with NSAID hypersensitivity has observed an increased incidence of chronic urticaria presenting years after the initial diagnosis. ${ }^{48}$ No other long-term follow-up or any follow- up of preschool-aged patients with NSAID hypersensitivity was identified by our review.

\section{Management}

As with all allergic reactions, the best approach to management is the avoidance of re-exposure. This should be easy in group 2 (single drug, specific) reactions as most drugs have reasonable alternatives. Unfortunately, the most common clinical presentation in this age group seems to be of the nonspecific cross-reactive type with immediate angioedema/urticaria of unpredictable severity. Cross-reactivity to acetaminophen in young children with hypersensitivity to ibuprofen was estimated by various investigators to be between 4 and $25 \% .^{15,49,50}$ In these children, there is no other approved medication for the treatment of fever or acute pain, and management varies between the use of nonapproved COX-2-specific medications, such as rofecoxib and celecoxib, and the use of physical measures such as keeping the room cool, encouraging the drinking of fluids, and sponging with lukewarm water. ${ }^{51,52}$

\section{Discussion}

Overall, the current classification of NSAID hypersensitivity reactions can also be applied to such reactions in children less than 6 years of age. However, no cases of hypersensitivity pneumonitis or meningitis in this age group were found in the literature reviewed, whereas several reported cases of a single drug causing fixed eruptions or toxic epidermal necrolysis, both most likely delayed-type hypersensitivity reactions, could not be fitted in the current classification scheme and could be added to the group 2 reactions.

The epidemiologic data available for this age group are scattered and difficult to integrate. The virtual disappearance of ASA from the pediatric formulary, except for severely limited indications starting from the 1980s, also limits the data on hypersensitivity reactions to ASA in young children to older publications and may explain why initial reports on

Table 3. Proposed Weight-Adjusted Oral Challenge Protocol for Suspected Cross-Reactive Hypersensitivity Reactions to NSAID in Children Up to $40 \mathrm{~kg}$

\begin{tabular}{lcccccc}
\hline Challenge Medication & $\begin{array}{c}\text { Challenge Dose } \\
(\mathrm{mg} / \mathrm{kg})\end{array}$ & $\begin{array}{c}\text { Advance Dose } \\
\text { Schedule }\end{array}$ & $\begin{array}{c}\text { Interval between } \\
\text { Challenges }(\mathrm{h})\end{array}$ & $\begin{array}{c}\text { Maximal Dose } \\
(\mathrm{mg} / \mathrm{kg})\end{array}$ & $\begin{array}{c}\text { Range of Reaction } \\
\text { Dose }(\mathrm{mg} / \mathrm{kg})\end{array}$ & $\begin{array}{c}\text { Usual Time to } \\
\text { Reaction }(\mathrm{h})\end{array}$ \\
\hline Aspirin & 2.5 & Repeat same & 1 & 10 & $2.5-10$ & $1-4$ \\
Ibuprofen & 2.5 & Repeat same & 1 & 10 & $5-10$ & $2-4$ \\
Paracetamol & 5 & Repeat same & 1 & 20 & $10-20$ & $2-4$ \\
\hline
\end{tabular}


ASA-exacerbated respiratory disease starting in early childhood are largely not supported by later publications. Ibuprofen-induced bronchospasm has been documented in about $2 \%$ of asthmatic children above 6 years of age ${ }^{53}$ in a laboratory challenge setting, and there are isolated case reports, but the clinical significance of this finding in the context of treating the febrile asthmatic child without a history of previous adverse reactions is still unclear.

In patients with classic indications for the use of ASA and other NSAIDs, for example, Kawasaki disease, rheumatic fever, and juvenile rheumatoid arthritis, few, if any, hypersensitivity reactions in young children have been published in recent years.

Acetaminophen seems to cause a similar spectrum of clinical hypersensitivity reactions in a proportion of young children with NSAID hypersensitivity. However, since acetaminophen is not an anti-inflammatory medication but is nevertheless a COX inhibitor, ${ }^{3}$ it may be appropriate to change the terminology of the reactions to "COX inhibitor hypersensitivity," with a slightly modified classification scheme, as detailed in Figure 3. This is also appropriate since we know that the relative COX inhibitory efficacy of the various preparations can predict with remarkable accuracy the in vivo challenge results ${ }^{15}$ and the in vitro results of some tests in cross-reactive patients. ${ }^{54}$ Also, most of the anti-inflammatory activity of the NSAID is secondary to the COX-2 inhibition, and this activity is only marginally relevant to the hypersensitivity reactions elicited, as proven by the relative safety of the COX-2-selective medications. ${ }^{51,55,56}$

Available data in adults $22,25,26,28,30$ and suggestive data in children seem to point to a significant genetic heterogeneity and therefore geographic and populationspecific variations in the prevalence, pathogenicity, and clinical manifestations of these hypersensitivity reactions.

Nevertheless, this review emphasizes that although the incidence of hypersensitivity reactions to the commonly used antipyretics in young children is low and urticaria/ angiodema, that is, "only skin deep" reactions, may predominate, respiratory and systemic reactions have been documented.

A strong association of NSAID hypersensitivity with atopy and clinical atopic disease is seen in this age group and in older patients, and this may explain the relative male predominance (as opposed to female predominance in adults) since atopic disease in young children also shows a somewhat increased prevalence in boys.

Since the general prevalence of NSAID hypersensitivity in the pediatric age group is low, it would be a significant advantage if diagnostic challenge protocols and diagnostic criteria could be developed and agreed on in an international consensus, facilitating the comparison and integration of developed data. In this context of very young children, in whom the difference in weight can easily be a factor of 10 , we would strongly suggest adapting a weight-related challenge protocol similar to the one described in Table 3.

Almost no data are available on the natural history of the pediatric disease, the pathogenesis of angioedema/ urticaria reactions, or the genetic factors associated with this early hypersensitivity. All of these areas require further research. Also lacking are management options of fever in young children with cross-reactive hypersensitivity to ibuprofen and acetaminophen at antipyretic doses.

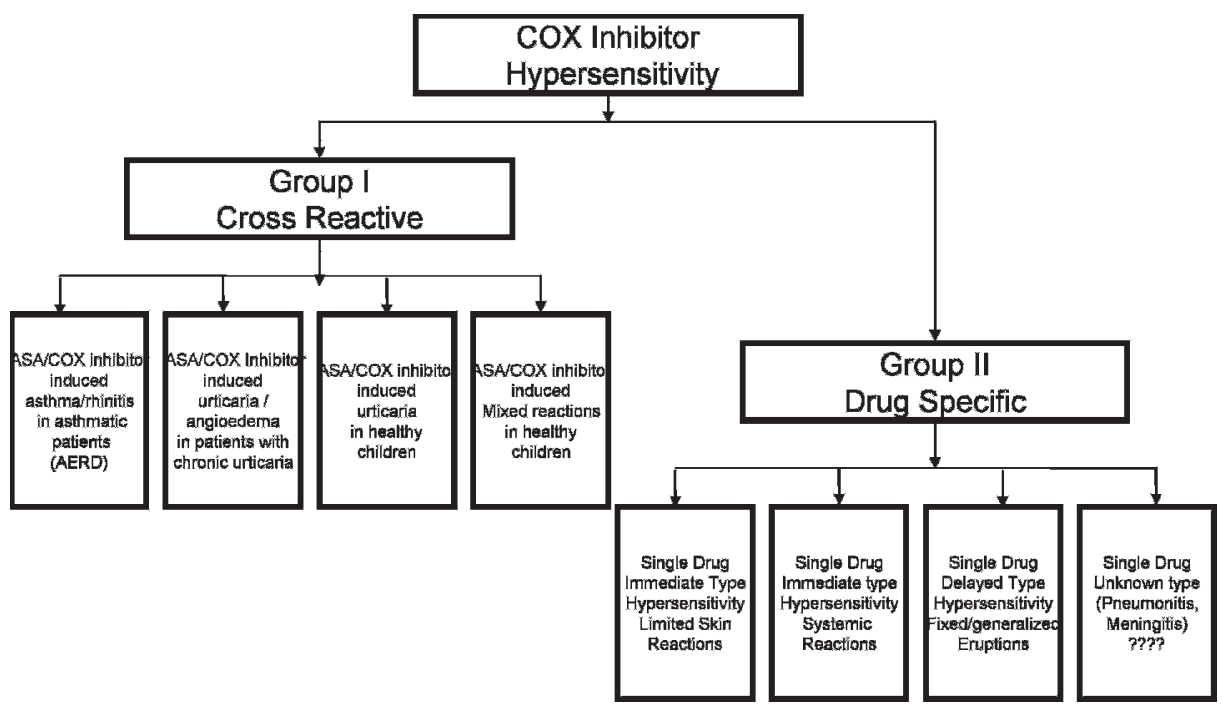

Figure 3. Proposed classification of cyclooxygenase inhibitor hypersensitivity reactions in preschool children. 


\section{Conclusion}

Although facial angioedema and urticaria are the most common manifestations of NSAID hypersensitivity in young children, systemic, cardiovascular, and respiratory symptoms have been documented.

The diagnosis usually requires an observed challenge with the implicated medication or medications in a protective environment performed by highly trained medical teams. Challenge procedures in young children need modification to adjust for age and weight, as well as different patterns of presentation.

Although reactions may be elicited during ASA or ibuprofen challenge in a small proportion of asthmatic children, the vast majority of asthmatics do not show associated respiratory exacerbations with antipyretic doses of ibuprofen. Physicians prescribing antipyretics for children should be aware, however, of the association of atopy and NSAID hypersensitivity even in preschool-aged children.

The incidence of acetaminophen hypersensitivity in children with NSAID-induced reactions is higher than expected for a random association, at least in some ethnic groups, and patients with documented reactions to ibuprofen may require a formal challenge before full doses of acetaminophen are prescribed.

\section{References}

1. Aspirin and Reye syndrome. Committee on Infectious Diseases. Pediatrics 1982;69:810-2.

2. Drug and Device Approvals - March 1995. 1995. U.S. Food and Drug administration, Center for Drug Evaluation and Research.

3. Chandrasekharan NV, Dai $\mathrm{H}$, Roos KL, et al. COX-3, a cyclooxygenase-1 variant inhibited by acetaminophen and other analgesic/antipyretic drugs: cloning, structure, and expression. Proc Natl Acad Sci U S A 2002;99:13926-31.

4. Stevenson DD, Simon RA, Zuraw BL. Sensitivity to Aspirin and Nonsteroidal Antiinflammatory Drugs. In: Elliott Middleton J, Charles E Reed, Elliot F. Ellis, N. Franklin Adkinson J, John W. Yunginger, William W Busse, editors. Adkinson: Middleton's Allergy, Principles and Practice. 6 ed. Mosby Inc.; 2003. p. 1695.

5. Settipane RA, Constantine HP, Settipane GA. Aspirin intolerance and recurrent urticaria in normal adults and children. Epidemiology and review. Allergy 1980;35:149-54.

6. Martinez-Mir I, Garcia-Lopez M, Palop V, et al. A prospective study of adverse drug reactions as a cause of admission to a paediatric hospital. Br J Clin Pharmacol 1996;42:319-24.

7. Kidon MI, See Y. Adverse drug reactions in Singaporean children. Singapore Med J 2004;45:574-7.

8. Titchen T, Cranswick N, Beggs S. Adverse drug reactions to nonsteroidal anti-inflammatory drugs, COX-2 inhibitors and paracetamol in a paediatric hospital. Br J Clin Pharmacol 2005; 59:718-23.
9. Vedanthan PK, Menon MM, Bell TD, Bergin D. Aspirin and tartrazine oral challenge: incidence of adverse response in chronic childhood asthma. J Allergy Clin Immunol 1977;60:8-13.

10. Schuhl JF, Pereyra JG. Oral acetylsalicylic acid (aspirin) challenge in asthmatic children. Clin Allergy 1979;9:83-8.

11. Rachelefsky GS, Coulson A, Siegel SC, Stiehm ER. Aspirin intolerance in chronic childhood asthma: Detected by oral challenge. Pediatrics 1975;56:443-8.

12. Falliers CJ. Aspirin and subtypes of asthma: risk factor analysis. J Allergy Clin Immunol 1973;52:141-7.

13. Capriles-Behrens E, Caplin J, Sanchez-Borges M. NSAID facial angioedema in a selected pediatric atopic population. J Investig Allergol Clin Immunol 2000;10:277-9.

14. Sanchez-Borges M, Capriles-Hulett A. Atopy is a risk factor for non-steroidal anti-inflammatory drug sensitivity. Ann Allergy Asthma Immunol 2000;84:101-6.

15. Kidon MI, Kang LW, Chin CW, et al. Early Presentation With Angioedema and Urticaria in Cross-reactive Hypersensitivity to Nonsteroidal Antiinflammatory Drugs Among Young, Asian, Atopic Children. Pediatrics 2005.

16. Speer F, Denison TR, Baptist JE. Aspirin allergy. Ann Allergy 1981; 46:123-6.

17. Lesko SM, Mitchell AA. The safety of acetaminophen and ibuprofen among children younger than two years old. Pediatrics 1999;104:e39.

18. Lesko SM, Louik C, Vezina RM, Mitchell AA. Asthma morbidity after the short-term use of ibuprofen in children. Pediatrics 2002; 109:E20.

19. Szczeklik A, Sladek K, Dworski R, et al. Bronchial aspirin challenge causes specific eicosanoid response in aspirin-sensitive asthmatics. Am J Respir Crit Care Med 1996;154:1608-14.

20. Cowburn AS, Sladek K, Soja J, et al. Overexpression of leukotriene C4 synthase in bronchial biopsies from patients with aspirinintolerant asthma. J Clin Invest 1998;101:834-46.

21. Sanak M, Simon HU, Szczeklik A. Leukotriene C4 synthase promoter polymorphism and risk of aspirin-induced asthma. Lancet 1997;350:1599-600.

22. Sanak M, Pierzchalska M, Bazan-Socha S, Szczeklik A. Enhanced expression of the leukotriene $\mathrm{C}(4)$ synthase due to overactive transcription of an allelic variant associated with aspirin-intolerant asthma. Am J Respir Cell Mol Biol 2000;23:290-6.

23. Yoshida S, Sakamoto H, Ishizaki Y, et al. Efficacy of leukotriene receptor antagonist in bronchial hyperresponsiveness and hypersensitivity to analgesic in aspirin-intolerant asthma. Clin Exp Allergy 2000;30:64-70.

24. Dahlen SE, Malmstrom K, Nizankowska E, et al. Improvement of aspirin-intolerant asthma by montelukast, a leukotriene antagonist: a randomized, double-blind, placebo-controlled trial. Am J Respir Crit Care Med 2002;165:9-14.

25. Van Sambeek R, Stevenson DD, Baldasaro M, et al. 5' flanking region polymorphism of the gene encoding leukotriene $\mathrm{C} 4$ synthase does not correlate with the aspirin-intolerant asthma phenotype in the United States. J Allergy Clin Immunol 2000;106: 72-6.

26. Choi JH, Kim SH, Bae JS, et al. Lack of an association between a newly identified promoter polymorphism $(-1702 \mathrm{G}>\mathrm{A})$ of the leukotriene C4 synthase gene and aspirin-intolerant asthma in a Korean population. Tohoku J Exp Med 2006;208:49-56. 
27. Oosaki R, Mizushima Y, Mita H, Shida T, Akiyama K, Kobayashi M. Urinary leukotriene E4 and 11-dehydrothromboxane B2 in patients with aspirin-sensitive asthma. Allergy 1997;52:470-3.

28. Akahoshi M, Obara K, Hirota $\mathrm{T}$, et al. Functional promoter polymorphism in the TBX21 gene associated with aspirin-induced asthma. Hum Genet 2005;117:16-26.

29. Dekker JW, Nizankowska E, Schmitz-Schumann M, et al. Aspirininduced asthma and HLA-DRB1 and HLA-DPB1 genotypes. Clin Exp Allergy 1997;27:574-7.

30. Choi JH, Lee KW, Oh HB, et al. HLA association in aspirinintolerant asthma: DPB $1 * 0301$ as a strong marker in a Korean population. J Allergy Clin Immunol 2004;113:562-4.

31. Szczeklik A. Aspirin-induced asthma as a viral disease. Clin Allergy 1988;18:15-20.

32. Tan Y, Collins-Williams C. Aspirin-induced asthma in children. Ann Allergy 1982;48:1-5.

33. Kauppinen $\mathrm{K}$, Juntunen $\mathrm{K}$, Lanki $\mathrm{H}$. Urticaria in children. Retrospective evaluation and follow-up. Allergy 1984;39:469-72.

34. Legrain V, Taieb A, Sage T, Maleville J. Urticaria in infants: a study of forty patients. Pediatr Dermatol 1990;7:101-7.

35. Moore-Robinson M, Warin RP. Effect of salicylates in urticaria. Br Med J 1967;4:262-4.

36. Wright AL, Minford A. Urticaria and hidden salicylates. Pediatr Dermatol 1999;16:463-4.

37. Botey J, Ibero $\mathrm{M}$, Malet $\mathrm{A}$, et al. Aspirin-induced recurrent urticaria and recurrent angioedema in non-atopic children. Ann Allergy 1984;53:265-7.

38. Diaz JM, Perez MA, Gracia Bara MT, et al. Allergic reactions due to ibuprofen in children. Pediatr Dermatol 2001;18:66-7.

39. de Almeida MA, Gaspar AP, Carvalho FS, et al. Adverse reactions to acetaminophen, ASA, and NSAIDs in children: what alternatives. Allergy Asthma Proc 1997;18:313-8.

40. Botey J, Navarro C, Aulesa C, et al. Acetyl salicylic acid inducedurticaria and/or angioedema in atopic children. Allergol Immunopathol (Madr ) 1988;16:43-7.

41. Kidon MI, Abramovitch I, Steinberg S, et al. Cross reactive hypersensitivity to COX inhibitors in a child with mild Allergic Rhinitis, a case report. IMAJ 2005;Accepted for publication.

42. Goraya JS, Virdi VS. To the editor: Exacerbation of asthma by ibuprofen in a very young child. Pediatr Pulmonol 2001;32:262.

43. Sehgal VN. Paracetamol-induced bilateral symmetric, multiple fixed drug eruption (MFDE) in a child. Pediatr Dermatol 1999;16: $165-6$.
44. Halevi A, Ben Amitai D, Garty BZ. Toxic epidermal necrolysis associated with acetaminophen ingestion. Ann Pharmacother 2000; 34:32-4.

45. Dogra S, Handa S. Bullous necrotizing fixed drug eruption in an infant. Pediatr Dermatol 2004;21:281-2.

46. Kanwar AJ, Bharija SC, Belhaj MS. Fixed drug eruptions in children: a series of 23 cases with provocative tests. Dermatologica 1986;172:315-8.

47. Aberer W, Bircher A, Romano A, et al. Drug provocation testing in the diagnosis of drug hypersensitivity reactions: general considerations. Allergy 2003;58:854-63.

48. Asero R. Intolerance to nonsteroidal anti-inflammatory drugs might precede by years the onset of chronic urticaria. J Allergy Clin Immunol 2003;111:1095-8.

49. Boussetta K, Ponvert C, Karila C, et al. Hypersensitivity reactions to paracetamol in children: a study of 25 cases. Allergy 2005;60: 1174-7.

50. Pastorello EA, Zara C, Riario-Sforza GG, et al. Atopy and intolerance of antimicrobial drugs increase the risk of reactions to acetaminophen and nimesulide in patients allergic to nonsteroidal anti-inflammatory drugs. Allergy 1998;53:880-4.

51. Gyllfors P, Bochenek G, Overholt J, et al. Biochemical and clinical evidence that aspirin-intolerant asthmatic subjects tolerate the cyclooxygenase 2 -selective analgetic drug celecoxib. J Allergy Clin Immunol 2003;111:1116-21.

52. Sanchez BM, Capriles-Hulett A, Caballero-Fonseca F, Perez CR. Tolerability to new COX-2 inhibitors in NSAID-sensitive patients with cutaneous reactions. Ann Allergy Asthma Immunol 2001;87: 201-4.

53. Debley JS, Carter ER, Gibson RL, et al. The prevalence of ibuprofen-sensitive asthma in children: a randomized controlled bronchoprovocation challenge study. J Pediatr 2005;147: 233-8.

54. Gray PA, Warner TD, Vojnovic I, et al. Effects of non-steroidal anti-inflammatory drugs on cyclo-oxygenase and lipoxygenase activity in whole blood from aspirin-sensitive asthmatics vs healthy donors. Br J Pharmacol 2002;137:1031-8.

55. Dahlen B, Szczeklik A, Murray JJ. Celecoxib in patients with asthma and aspirin intolerance. The Celecoxib in AspirinIntolerant Asthma Study Group. N Engl J Med 2001;344:142.

56. Garcia-Rodriguez RM, Hinojosa M, Camacho-Garrido E, et al. Celecoxib, safe in NSAID intolerance. Allergy 2002;57:1085-6. 